\title{
Study on Millimeter-Wave Vivaldi Rectenna and Arrays with High Conversion Efficiency
}

\author{
Guan-Nan Tan, ${ }^{1}$ Xue-Xia Yang, ${ }^{1,2}$ Huan Mei, ${ }^{1}$ and Zhong-Liang Lu ${ }^{1,3}$ \\ ${ }^{1}$ School of Communications and Information Engineering, Shanghai University, Shanghai 200072, China \\ ${ }^{2}$ Key Laboratory of Specialty Fiber Optics and Optical Access Networks, Shanghai University, Shanghai 200072, China \\ ${ }^{3}$ Jiangxi University of Science and Technology, Jiangxi 341000, China
}

Correspondence should be addressed to Xue-Xia Yang; yang.xx@shu.edu.cn

Received 11 May 2016; Accepted 26 October 2016

Academic Editor: Herve Aubert

Copyright (C) 2016 Guan-Nan Tan et al. This is an open access article distributed under the Creative Commons Attribution License, which permits unrestricted use, distribution, and reproduction in any medium, provided the original work is properly cited.

\begin{abstract}
A novel Vivaldi rectenna operated at $35 \mathrm{GHz}$ with high millimeter wave to direct current (MMW-to-DC) conversion efficiency is presented and the arrays are investigated. The measured conversion efficiency is $51.6 \%$ at $35 \mathrm{GHz}$ and the efficiency higher than $30 \%$ is from $33.2 \mathrm{GHz}$ to $36.6 \mathrm{GHz}$ when the input MMW power is $79.4 \mathrm{~mW}$. The receiving Vivaldi antenna loaded with metamaterial units has a high gain of $10.4 \mathrm{dBi}$ at $35 \mathrm{GHz}$. A SIW- (substrate integrated waveguide-) to-microstrip transition is designed not only to integrate the antenna with the rectifying circuit directly but also to provide the DC bypass for the rectifying circuit. When the power density is $8.7 \mathrm{~mW} / \mathrm{cm}^{2}$, the received MMW power of the antenna is $5.6 \mathrm{~mW}$, and the maximum conversion efficiency of the rectenna element is $31.5 \%$. The output DC voltage of the element is nearly the same as that of the parallel array and is about half of the series array. The DC power obtained by the $1 \times 2$ rectenna arrays is about two times as much as that of the element. The conversion efficiencies of the arrays are very close to that of the element. Large scale arrays could be expended for collecting more DC power.
\end{abstract}

\section{Introduction}

WPT (wireless power transmission) technology is used to deliver electric power without wires. It can date back to 1960s when Brown carried out the first WPT experiment [1]. To solve the energy crises, the concept of space-based SSP (space solar power) was conceived by Dr. Peter Glaser in 1968 [2], promoting the development of WPT technology [3, 4]. Compared to the microwave WPT systems, the millimeter-wave systems have the advantages of smaller size and higher system transmission efficiency for long distance WPT, which can be applied in the space where there is no atmosphere loss [5]. The rectenna, consisting of a receiving antenna and a rectifying circuit, plays an important role in the WPT systems.

Many rectennas at microwave bands have been developed [6-11], and the RF-to-DC conversion efficiency can reach about $80 \%$ when the rectifying circuits obtain about $100 \mathrm{~mW}$ input power. Until now, only a few millimeter-wave rectennas have been reported [12-16], which are listed in Table 1. The reported CPS (coplanar strip line) rectenna and patch rectenna operated at $35 \mathrm{GHz}$ obtain the measured conversion efficiencies of $38 \%$ and $29 \%$, respectively, when the input MMW power is $120 \mathrm{~mW}[12,13]$. To obtain the enough input power for the rectifying circuit, the transmitted millimeterwave power is high for the above two rectennas because of the low gain of the receiving antennas. In order to lessen the transmitting power demand and supply the required input power for the rectifying circuit, the $1 \times 2$ antenna array is designed for the rectifying circuit [14]. The rectenna element and the $2 \times 2$ series array obtain an efficiency of $35 \%$ when the power density is $30 \mathrm{~mW} / \mathrm{cm}^{2}$. However, the length of the rectifying circuit is longer than $2 \lambda_{0}$. Reference [15] reports a dual-band rectenna operated at 35 and $94 \mathrm{GHz}$ with the conversion efficiency of $53 \%$ and $37 \%$, respectively, under the power density of $30 \mathrm{~mW} / \mathrm{cm}^{2}$. The circuit is based on the CMOS $0.13-\mu \mathrm{m}$ technology, which is more expensive and complex to design comparing to the standard $\mathrm{PCB}$ technology. In [16], the voltage doubling rectifying circuit obtains measured conversion efficiency of $34 \%$ at $35 \mathrm{GHz}$ when the input power is $20 \mathrm{~mW}$. 
TABLE 1: Performances of the reported rectifying circuits/rectennas and this work.

\begin{tabular}{|c|c|c|c|c|c|}
\hline References & $f(\mathrm{GHz})$ & Diode connect & $P_{\text {in }}(\mathrm{mW})$ rectifying circuit & $P_{d}\left(\mathrm{~mW} / \mathrm{cm}^{2}\right)$ rectenna & eff $(\%)$ \\
\hline [12] & 35 & Parallel & 120 & - & 38 \\
\hline [13] & 35 & Series & 120 & - & 29 \\
\hline$[14]$ & 35 & Parallel & - & 30 & 35 \\
\hline$[15]$ & $\begin{array}{l}35 \\
94\end{array}$ & Parallel & - & 30 & $\begin{array}{l}53 \\
37\end{array}$ \\
\hline [16] & 35 & Doubling & 20 & - & 34 \\
\hline \multirow[t]{2}{*}{ This work } & \multirow[t]{2}{*}{35} & \multirow[t]{2}{*}{ Series } & $\begin{array}{c}20 \\
79.4\end{array}$ & - & $\begin{array}{l}43.7 \\
51.6\end{array}$ \\
\hline & & & - & 8.7 & 31.5 \\
\hline
\end{tabular}

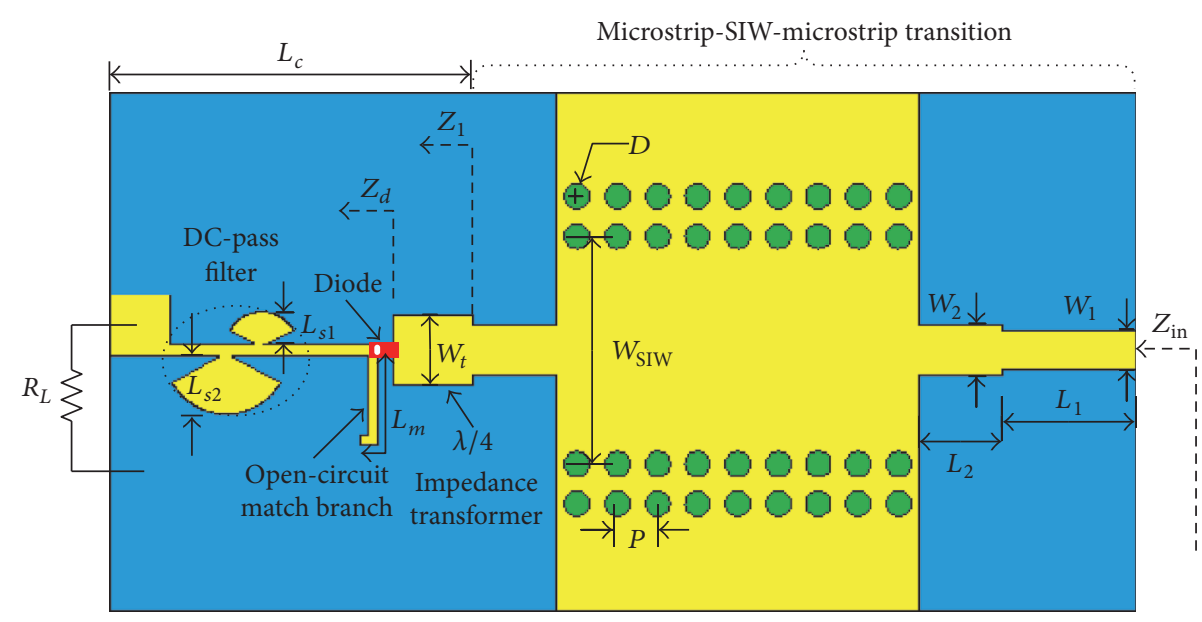

FIGURE 1: Structure of the rectifying circuit.

In this paper, a novel Vivaldi rectenna operated at $35 \mathrm{GHz}$ with high MMW-to-DC conversion efficiency is presented and the array design is investigated. The rectifying circuit adopts the series-mounted diode topological structure and has small and simple structure. The receiving antenna is a broadband end-fire Vivaldi antenna with high gain and harmonic suppression function and could be integrated directly to the rectifying circuit to form the rectenna. The performances of the rectifying circuit, rectenna element, and arrays are verified by the measurements. The rectenna element has high conversion efficiency and compact structure and could be extended to large arrays. Table 1 summarizes the performances of the reported rectifying circuits or rectennas and this work.

\section{Rectenna Design}

2.1. Rectifying Circuit Design. To design the rectifying circuit, it is essential to determine the diode's input impedance. At microwave band, the diode's input impedance could be obtained from the closed-form equation [6, 7] or the commercial software of ADS (Advanced Design System) and AWR Microwave Office [8-11], in which the parameters of the diode model provided by the datasheet are accurate. However, at millimeter-wave bands, the equivalent circuit parameters of the diode model are inaccurate and the simulated results will not be precise enough, so the conversion efficiencies of the designed rectifying circuits are low [12-14, 16]. In this study, the proposed rectifying circuit is designed by the experimental method.

2.1.1. Rectifying Circuit Configuration. The geometrical structure of the proposed rectifying circuit operating at $35 \mathrm{GHz}$ is shown in Figure 1, which consists of a diode in series configuration, an open-circuit match branch, a quarter-wave microstrip line impedance transformer, a microstrip-SIWmicrostrip transition, two radial stubs, and a resistance load $R_{L}$. The resistive load $R_{L}$ is used to collect the DC power. The rectifying circuit is designed on Rogers 5880 substrate with the relative permittivity of 2.2 , the loss tangent of 0.0009 , and the thickness of $0.254 \mathrm{~mm}$.

The commercial Schottky diode of MA4E1317 is used for rectifying. The diode has the forward and breakdown voltages of $0.7 \mathrm{~V}$ and $7 \mathrm{~V}$, respectively. The zero-bias junction capacitance $C_{j}$ is $0.02 \mathrm{pF}$ and the series resistance is $4 \Omega$. The diode's input impedance $Z_{d}$ is a complex due to the junction capacitance $C_{j}$. The open-circuit match branch with the length of $L_{m}$ is inserted between the diode and the DCpass filter to eliminate the imaginary part of the diode's input impedance $Z_{d}$. The quarter-wave microstrip line impedance 
TABLE 2: Dimensions of the rectifying circuit and the microstrip-SIW-microstrip transition.

\begin{tabular}{lccccc}
\hline$L_{m}$ & $W_{t}$ & $L_{s 1}$ & $L_{s 2}$ & $L_{c}$ & $W_{\text {SIW }}$ \\
2.02 & 1.4 & 0.6 & 1.12 & 4.55 \\
\hline$P$ & $D$ & $W_{1}$ & $W_{2}$ & $L_{1}$ & $L_{2}$ \\
0.8 & 0.5 & 0.78 & 1 & 3.1 & 1.55 \\
\hline
\end{tabular}

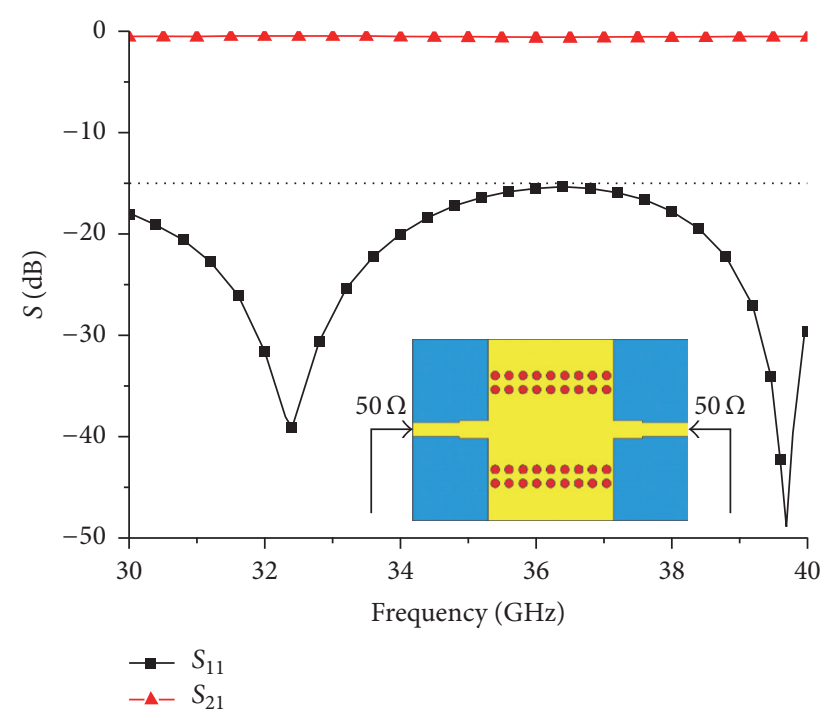

FIGURE 2: Simulated $S$-parameters of the microstrip-SIW-microstrip transition.

transformer with the width of $W_{t}$ is used to convert the real part of $Z_{d}$ into $Z_{1}$, which is designed to be $50 \Omega$.

To integrate the rectifying circuit with the following SIW receiving Vivaldi antenna, the microstrip-SIW-microstrip transition with zero-phase shift is designed. With this transition, the input impedance and the conversion efficiency of the proposed rectifying circuit can be measured in experiment. The vias in SIW also provide the DC bypass to collect the DC power because of the series configuration of the diode. The simulated $S$-parameters of the microstrip-SIW-microstrip transition are shown in Figure 2. It can be observed that the reflection coefficient $\left|S_{11}\right|$ is less than $-15 \mathrm{~dB}$ and the insert loss $\left|S_{21}\right|$ is lower than $0.6 \mathrm{~dB}$ within the bandwidth from $30 \mathrm{GHz}$ to $40 \mathrm{GHz}$, which ensures the good passband performance of the transition. Meanwhile, the simulated phase shift of the designed transition at $35 \mathrm{GHz}$ is about $0^{\circ}$, which indicates that the input impedance $Z_{\text {in }}$ of the rectifying circuit shown in Figure 1 is equal to $Z_{1}$.

The two radial stubs with the lengths of $L_{s 1}$ and $L_{s 2}$ are designed as a DC-pass filter to prevent the unwanted fundamental wave and the second harmonic from passing the resistive load $R_{L}$. The simulated $S$ parameters of the DC-pass filter are shown in Figure 3. It can be found that the filter shows good DC-pass performance with the attenuations of $35 \mathrm{~dB}$ and $38 \mathrm{~dB}$ at $35 \mathrm{GHz}$ and $70 \mathrm{GHz}$, respectively.

2.1.2. Rectifying Circuit Experiments. The exact sizes of the high efficiency rectifying circuit shown in Figure 1 are determined by the experimental method. The two parameters, the

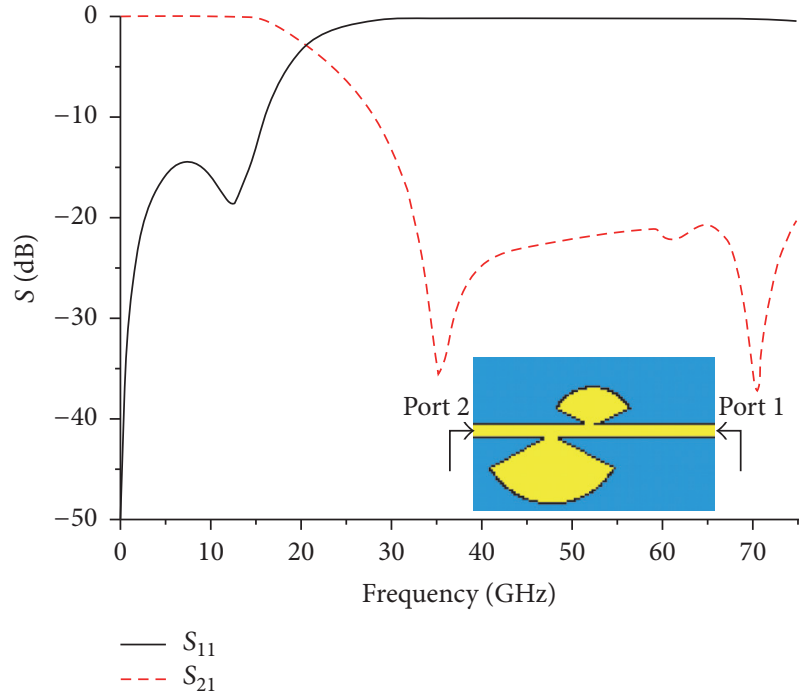

FIgURE 3: Simulated $S$-parameters of the DC-pass filter.

length $L_{m}$ of the open-circuit match branch and the width $W_{t}$ of the quarter wavelength transformer, are optimized to cancel the imaginary part of the diode's input impedance $Z_{d}$ and match the real part of $Z_{d}$ to $50 \Omega$. Several circuits with the same length $L_{m}$ and different width $W_{t}$ are fabricated. The initial length $L_{m}$ is $\lambda_{g} / 2$, and $\lambda_{g}$ is the waveguide wavelength in the microstrip line. To eliminate the imaginary part of the input impedance $Z_{\text {in }}$ of the rectifying circuit, the length of $L_{m}$ in each circuit is adjusted. When the input impedance $Z_{\text {in }}$ is matched to $50 \Omega$, which is measured by the Agilent VNA (Vector Network Analyzer) of N5227A, the sizes of $L_{m}$ and $W_{t}$ are determined, and the high efficiency rectifying circuit is obtained. The final dimensions of the rectifying circuit are given in Table 2. The measured reflection coefficient $\left|S_{11}\right|$ versus frequency is shown in Figure 4. It can be seen that when the input power is $10 \mathrm{dBm}$ and the load is $550 \Omega$, the measured $\left|S_{11}\right|$ is $-19.6 \mathrm{~dB}$ at $35 \mathrm{GHz}$ and the corresponding $Z_{\text {in }}$ is $(46+j 6.7) \Omega$ which can be found in the Smith chart of the VNA. The diode's input impedance $Z_{d}$ calculated from $Z_{\text {in }}$ is $(22.7-j 3.3) \Omega$. It is observed that the imaginary part of $Z_{d}$ is small and the open-circuit match branch works well.

The MMW-DC conversion efficiency $\eta$ of the rectifying circuit can be calculated by

$$
\eta=\frac{V_{\mathrm{dc}}}{R_{L} P_{\text {in }}} \times 100 \%
$$

where $R_{L}$ is the resistance load, $V_{\mathrm{dc}}$ is the output DC voltage on the load that can be read by a voltmeter, and $P_{\text {in }}$ is the input MMW power of the circuit. When the input power is 


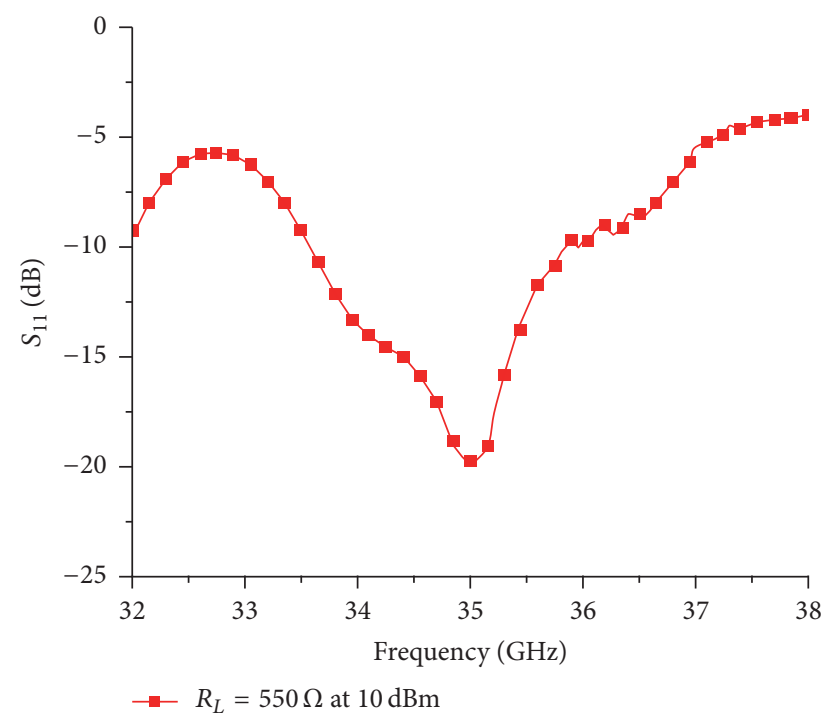

FIgURE 4: Measured reflection coefficient $\left|S_{11}\right|$ versus frequency, $P_{\text {in }}=10 \mathrm{dBm}$, and $R_{L}=550 \Omega$.

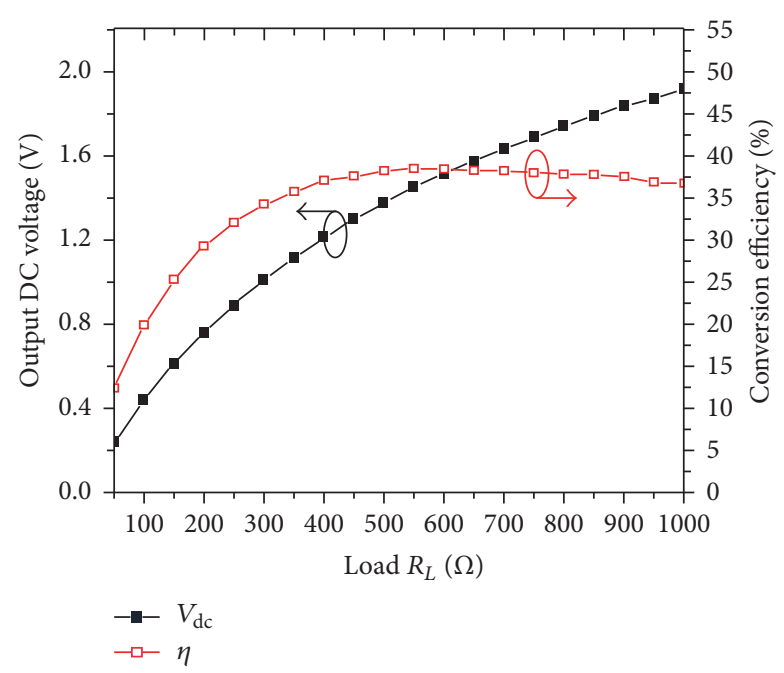

FIGURE 5: Measured output DC voltages and conversion efficiencies versus load $R_{L}, P_{\text {in }}=10 \mathrm{dBm}$, and $f=35 \mathrm{GHz}$.

$10 \mathrm{dBm}$, the measured output DC voltages and the conversion efficiencies versus the load at $35 \mathrm{GHz}$ are shown in Figure 5. It can be seen that the conversion efficiency increases with the load and gets the peak of $38.6 \%$ at $550 \Omega$. When the load keeps on increasing, the output DC voltage continues to increase while the efficiency decreases. So the optimal load resistance is $550 \Omega$.

When the load resistance is $550 \Omega$, the measured output DC voltages and conversion efficiencies versus the input power are plotted in Figure 6. It is found that the output DC voltage and the conversion efficiency increase with the input power. When the input power is $19 \mathrm{dBm}(79.4 \mathrm{~mW})$, the output voltage and conversion efficiency are $4.75 \mathrm{~V}$ and $51.6 \%$, respectively. When the input power continues to increase, the maximum voltage will exceed the breakdown voltages and the conversion efficiency decreases. The conversion efficiency

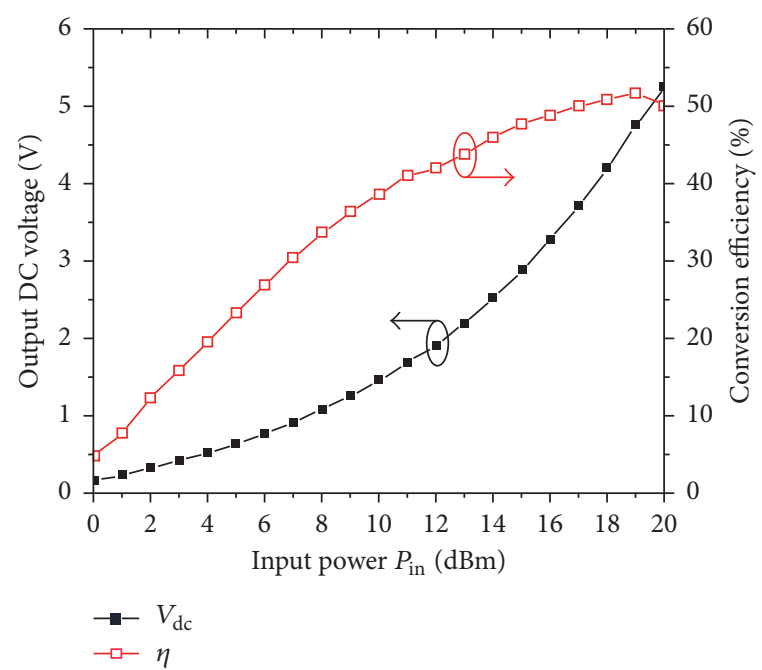

FIGURE 6: Measured output DC voltages and conversion efficiencies versus input power, $R_{L}=550 \Omega$, and $f=35 \mathrm{GHz}$.

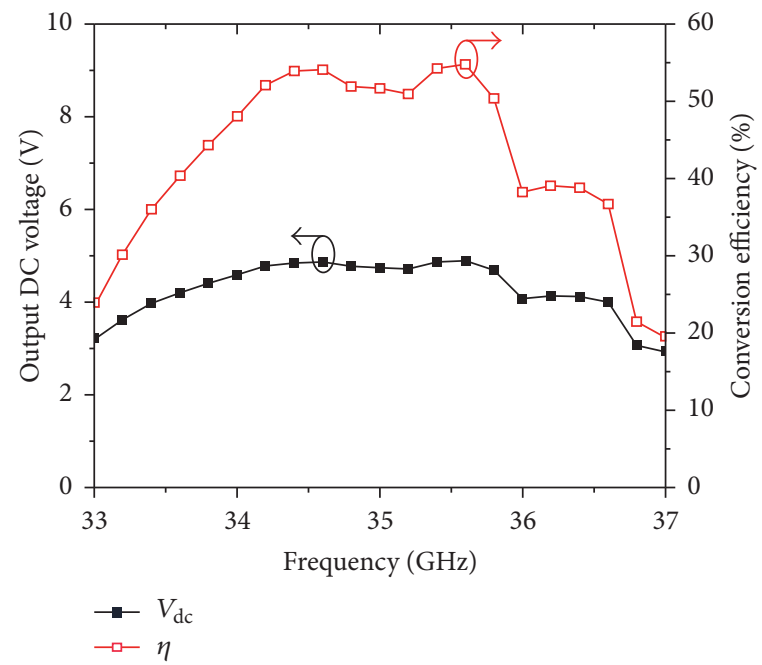

FIGURE 7: Measured output DC voltage and conversion efficiency versus frequency, $P_{\text {in }}=19 \mathrm{dBm}$, and $R_{L}=550 \Omega$.

is higher than $30 \%$ when the input power is higher than $7 \mathrm{dBm}(5 \mathrm{~mW})$. Figure 7 shows the measured output DC voltages and conversion efficiencies versus the frequency at the input power of $19 \mathrm{dBm}$ when the load resistance is $550 \Omega$. The efficiencies are all higher than $30 \%$ from 33.2 $\mathrm{GHz}$ to $36.6 \mathrm{GHz}$, which exhibits a relatively broadband performance.

2.2. Receiving Antenna Design. A novel Vivaldi slot is used as the receiving antenna, which is shown in Figure 8. It has the characteristics of high gain, broad bandwidth, and harmonic suppression. The Vivaldi slot is excited by a SIW, which is formed by two extra rows of metal vias to bound the energy within the SIW tightly and enhance the transmission performance. The center-symmetric metamaterial units with zero effective permittivity at $35 \mathrm{GHz}$ are loaded on the 


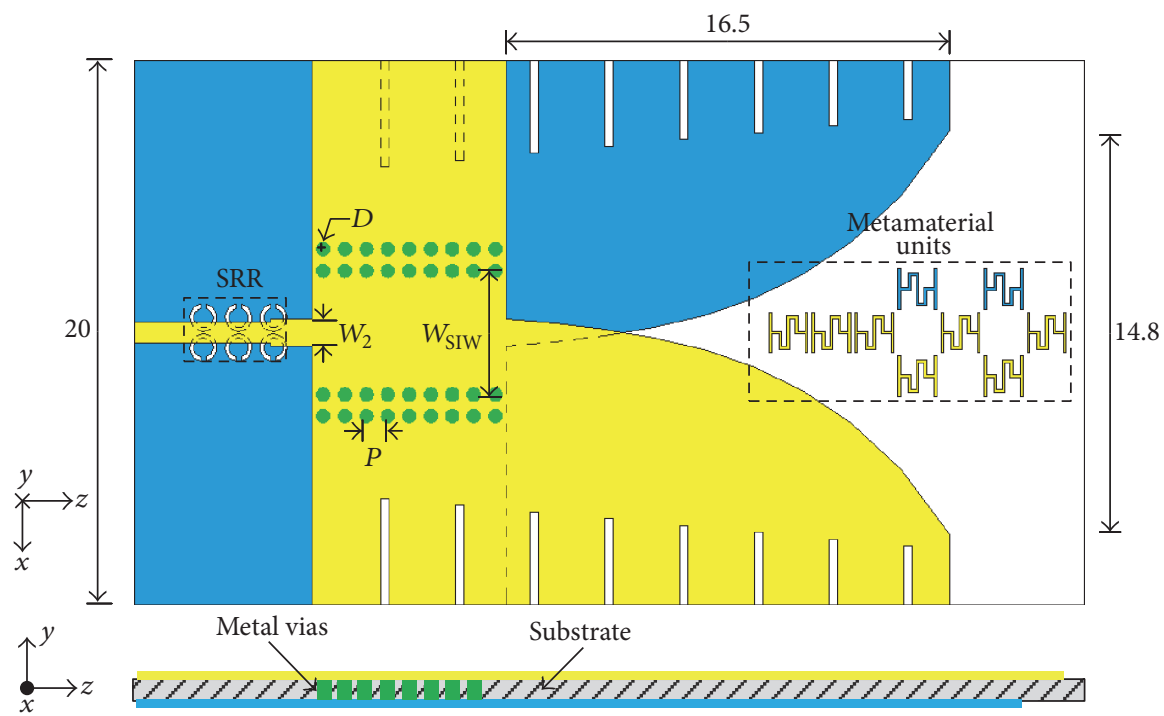

FIGURE 8: Structure of receiving antenna (units: $\mathrm{mm}$ ).

Vivaldi slot to improve the antenna gain. The microstripto-SIW transformer is designed to feed the antenna, and the characteristic impedance of the microstrip line is $50 \Omega$. In order to prevent the second harmonics generated by the nonlinear diode from reradiating by the antenna, the SRR (split ring resonator) units are etched on the ground of the microstrip feed line to act as a low pass filter. The substrate used for the receiving antenna is the same as that for the rectifying circuit.

The simulated and measured reflection coefficients and gains of the proposed Vivaldi antenna are plotted in Figure 9. The antenna exhibits a broad bandwidth of 38.5\% ranging from $26.5 \mathrm{GHz}$ to $40 \mathrm{GHz}$ with the reflection coefficient less than $-10 \mathrm{~dB}$. The gain varies from $9.3 \mathrm{dBi}$ to $10.4 \mathrm{dBi}$ within the Ka band and the highest gain is $10.4 \mathrm{dBi}$ at $35 \mathrm{GHz}$.

\section{Measurement of Rectenna and Arrays}

The rectifying circuit and the receiving antenna are integrated directly because they are all fed by the microstrip lines with the input impedances of $50 \Omega$. Figure 10 shows the structure of the proposed rectenna. The SIW in the receiving antenna is used not only to feed the Vivaldi slot but also to provide the DC bypass to the rectifying circuit so the rectenna structure is simplified. The total length $L_{c}$ of the rectifying circuit is $7 \mathrm{~mm}$, which is only about $0.82 \lambda_{0}$. The photographs of the fabricated rectenna are given in Figure 11.

The conversion efficiency of the rectenna is also calculated by (1), while $P_{\text {in }}$ is the MMW power received by the antenna. The output DC voltage $V_{\mathrm{dc}}$ is also read by a voltmeter. According to the Friis transmission equation, $P_{\text {in }}$ is calculated by

$$
P_{\text {in }}=\left(\frac{\lambda_{0}}{4 \pi r}\right)^{2} P_{t} G_{t} G_{r}
$$

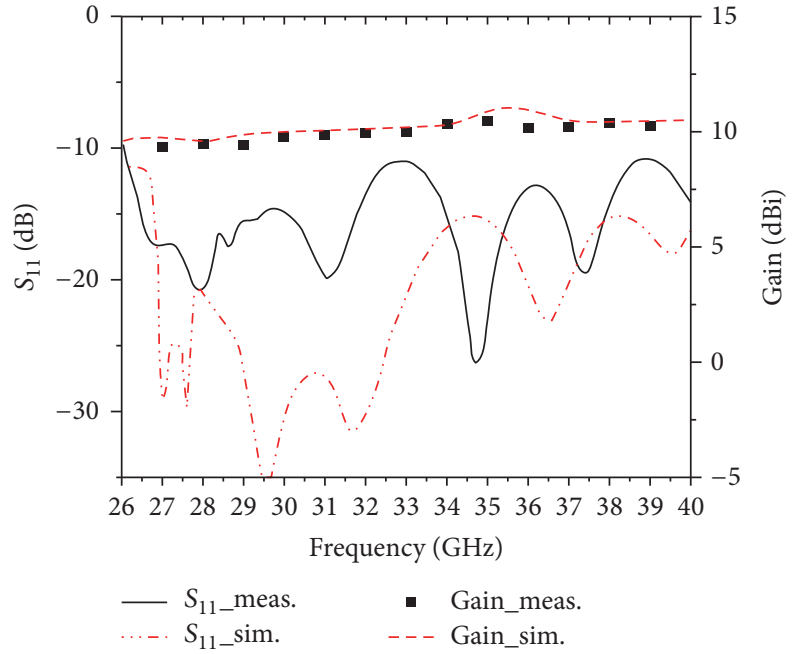

FIGURE 9: Simulated and measured reflection coefficients and gains of the receiving Vivaldi antenna.

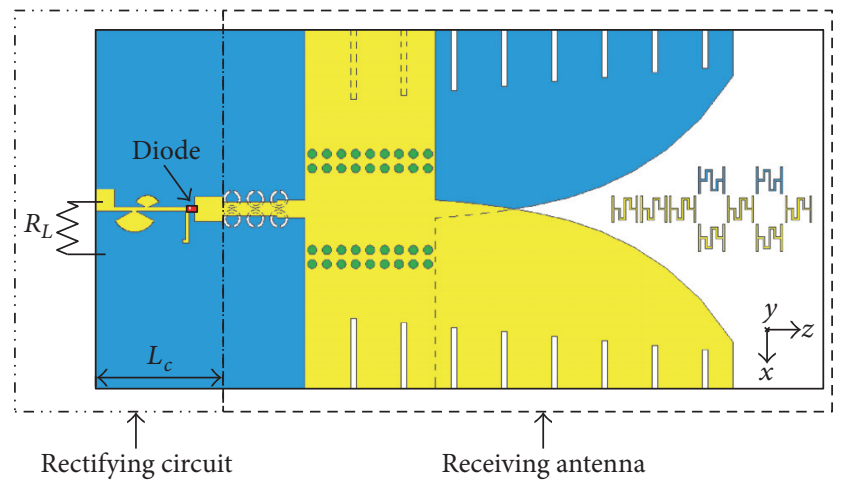

FIGURE 10: Structure of the proposed rectenna. 


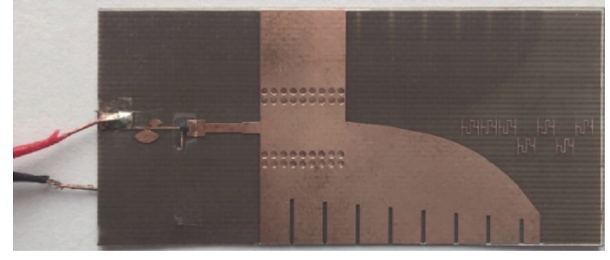

(a) Top view

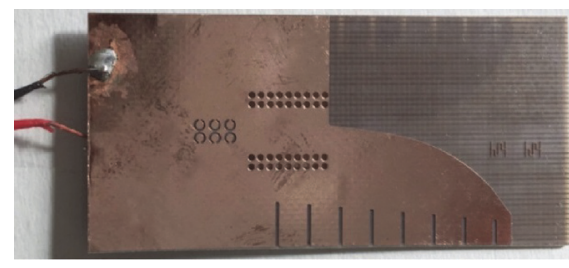

(b) Bottom view

FIGURE 11: Photograph of the fabricated rectenna.

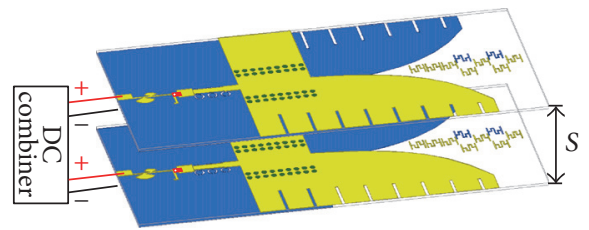

(a) Configuration of $1 \times 2$ rectenna array

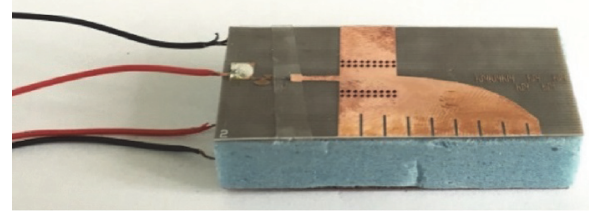

(b) Photograph of fabricated $1 \times 2$ rectenna array

Figure 12: $1 \times 2$ rectenna arrays.

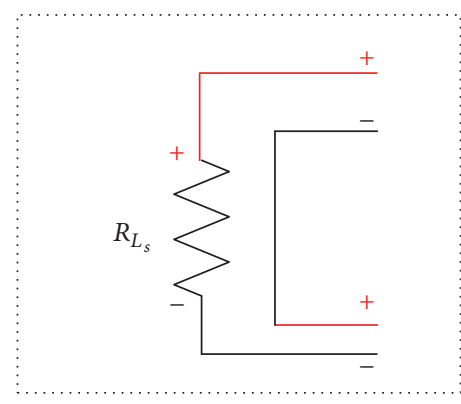

(a) Series layout

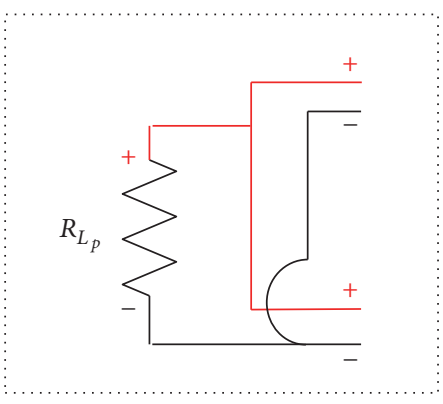

(b) Parallel layout

FIGURE 13: The series and parallel DC combiner circuits.

where $\lambda_{0}$ is the free space wavelength, $r$ is the propagation distance, and $P_{t}$ is the transmitting MMW power. The MMW signal source is Agilent E8257D. The MMW power is amplified by the amplifier with the saturation power of $36 \mathrm{dBm}$ at $35 \mathrm{GHz}$. A horn is used as the transmitting antenna, whose gain $G_{t}$ is $20.7 \mathrm{dBi}$ at $35 \mathrm{GHz}$. The measured gain of the receiving antenna is $G_{r}$ of $10.4 \mathrm{dBi}$. The rectenna is fixed at the distance of $r=58 \mathrm{~cm}$, and the rectenna is in the far field region.

In order to collect more DC power, rectenna arrays could be designed. The configuration of the $1 \times 2$ rectenna array is shown in Figure 12(a) and the photograph of the fabricated one is given in Figure 12(b). The space $S$ between the two rectenna elements is $8.5 \mathrm{~mm}\left(\approx \lambda_{0}\right.$.). The series and parallel DC combiner circuits are shown in Figure 13. The load resistances of the series and the parallel arrays are $R_{L_{s}}$ and $R_{L_{p}}$, which is calculated by $R_{L_{s}}=2 \cdot R_{L}=1100 \Omega$ and $R_{L_{p}}=R_{L} / 2=275 \Omega$, respectively. The rectenna element and the arrays are measured at $35 \mathrm{GHz}$.

The measured output DC voltage, DC power and the MMW-to-DC conversion efficiency of the rectenna element, and series and parallel arrays versus the power density $P_{d}$ are shown in Figures 13-14. Due to the limited MMW output power of the amplifier and the measurement system errors, the maximum power density is only $8.7 \mathrm{~mW} / \mathrm{cm}^{2}$ at the far field of $58 \mathrm{~cm}$. From Figure 14, it is observed that the output DC voltages increase with the power density. When the power density $P_{d}$ is $8.7 \mathrm{~mW} / \mathrm{cm}^{2}$, the output DC voltages of the rectenna element and parallel-connected and seriesconnected rectenna arrays are $0.99 \mathrm{~V}, 0.93 \mathrm{~V}$, and $1.87 \mathrm{~V}$, respectively. The output DC voltages of the rectenna element are very close to the parallel-connected rectenna array and are about half of the series-connected rectenna array.

As shown in Figure 15, the rectenna arrays obtain nearly double DC power comparing to the element, and the conversion efficiencies of the arrays and the element are nearly the same. When the power density $P_{d}$ is $8.7 \mathrm{~mW} / \mathrm{cm}^{2}$, the received MMW power of the antenna element is $5.6 \mathrm{~mW}$ and the maximum conversion efficiency of the element is $31.5 \%$ while that of the arrays is about $4 \%$ less. The differences of the conversion efficiencies are mainly caused by the fabrication and measurement errors. It can be predicable that the conversion efficiencies of the rectenna element and arrays will continue to increase with the power density. From the 


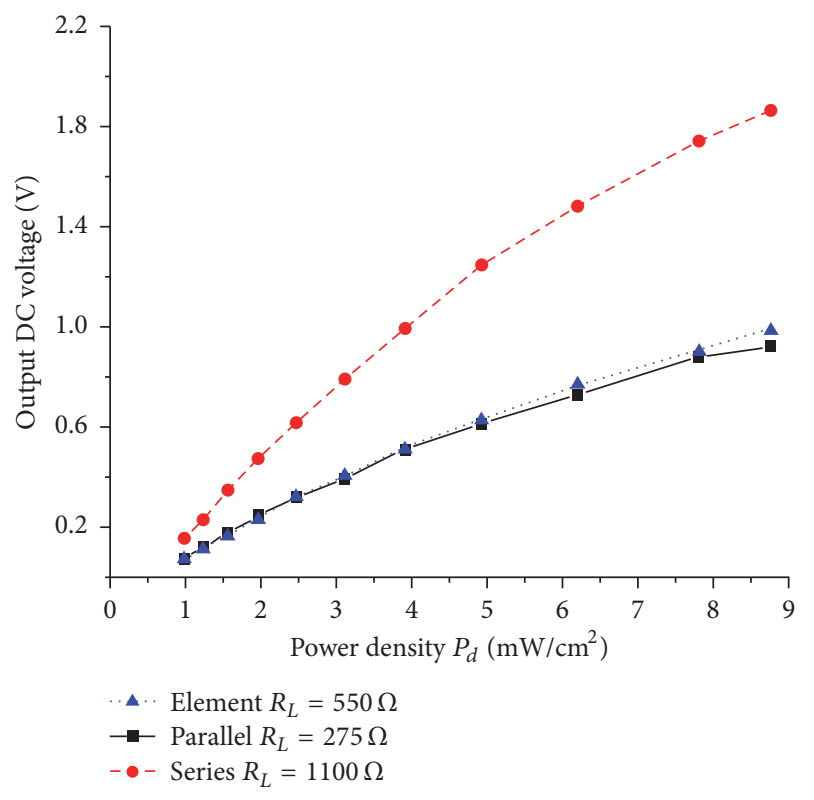

Figure 14: Measured output DC voltage.

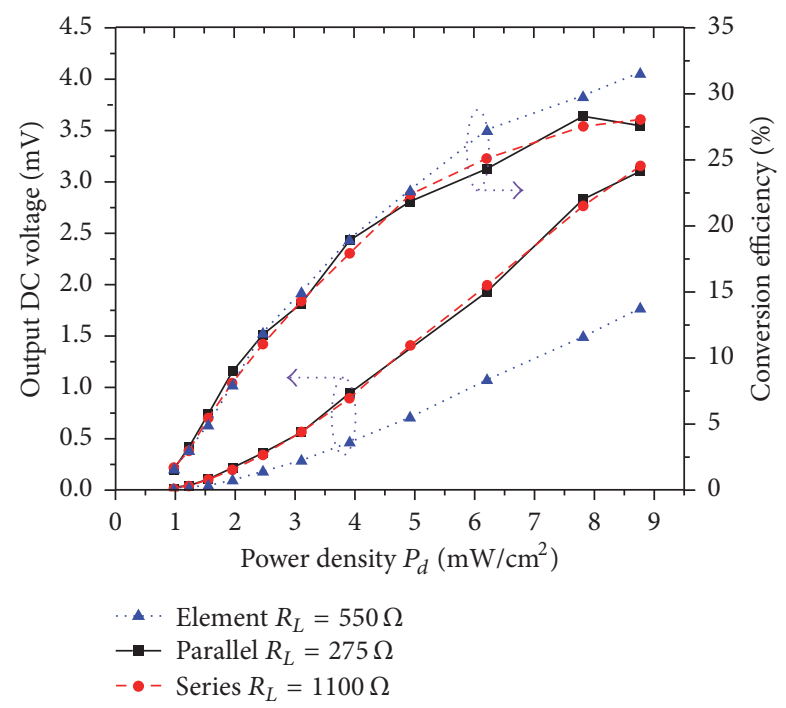

FIGURE 15: Measured output DC power and conversion efficiency.

measured results, it can be concluded that the rectenna arrays show good array performances and large scale arrays could be expanded to obtain more DC power.

\section{Conclusion}

In this paper, a novel high efficiency rectenna operated at $35 \mathrm{GHz}$ is proposed. The rectifying circuit exhibits a measured MMW-to-DC conversion efficiency higher than $30 \%$ from $33.2 \mathrm{GHz}$ to $36.6 \mathrm{GHz}$ when the input MMW power is $79.4 \mathrm{~mW}$, and the peak efficiency reaches $51.6 \%$ at $35 \mathrm{GHz}$. The receiving antenna with broad bandwidth, harmonic suppression, and high gain is integrated directly to the rectifying circuit to form the rectenna. The rectenna element and series-connected and parallel-connected rectenna arrays have been measured and the maximum conversion efficiency of $31.5 \%$ is obtained at a low power density of $8.7 \mathrm{~mW} / \mathrm{cm}^{2}$. The efficiency would increase under a higher power density condition. Compared to the rectenna element, the $1 \times 2$ arrays obtain nearly double DC power. The developed rectenna has the merits of low profile, small size, and low cost. It is easy to form large scale rectenna arrays to collect more MMW power suitable for millimeter-wave energy harvesting and wireless power transmission applications.

\section{Competing Interests}

The authors declare that there is no conflict of interests regarding the publication of this paper.

\section{Acknowledgments}

This work was supported by Natural Science Foundation of China under Grant no. 61271062.

\section{References}

[1] W. C. Brown, "Free-space transmission," IEEE Spectrum, vol. 1, no. 10, pp. 86-91, 1964.

[2] P. E. Glaser, "Power from the sun: its future," Science, vol. 162, no. 3856, pp. 857-861, 1968.

[3] W. C. Brown, "Satellite power stations: a new source of energy?" IEEE Spectrum, vol. 10, no. 3, pp. 38-47, 1973.

[4] W. C. Brown, "The technology and application of free-space power transmission by microwave beam," Proceedings of the IEEE, vol. 62, no. 1, pp. 11-25, 1974.

[5] B. Strassner and K. Chang, "Microwave power transmission: historical milestones and system components," Proceedings of the IEEE, vol. 101, no. 6, pp. 1379-1396, 2013.

[6] J. A. G. Akkermans, M. C. Van Beurden, G. J. N. Doodeman, and H. J. Visser, "Analytical models for low-power rectenna design," IEEE Antennas and Wireless Propagation Letters, vol. 4, no. 1, pp. 187-190, 2005.

[7] W.-H. Tu, S.-H. Hsu, and K. Chang, "Compact 5.8-GHz rectenna using stepped-impedance dipole antenna," IEEE Antennas and Wireless Propagation Letters, vol. 6, pp. 282-284, 2007.

[8] X.-X. Yang, C. Jiang, A. Z. Elsherbeni, F. Yang, and Y.-Q. Wang, "A novel compact printed rectenna for data communication systems," IEEE Transactions on Antennas and Propagation, vol. 61, no. 5, pp. 2532-2539, 2013.

[9] F. Zhang, X. Liu, F.-Y. Meng et al., "Design of a compact planar rectenna for wireless power transfer in the ISM band," International Journal of Antennas and Propagation, vol. 2014, Article ID 298127, 9 pages, 2014.

[10] S.-T. Khang, J. W. Yu, and W.-S. Lee, "Compact folded dipole rectenna with RF-based energy harvesting for IoT smart sensors," Electronics Letters, vol. 51, no. 12, pp. 926-928, 2015.

[11] V. Kuhn, C. Lahuec, F. Seguin, and C. Person, "A multi-band stacked RF energy harvester with RF-to-DC efficiency up to 84\%," IEEE Transactions on Microwave Theory and Techniques, vol. 63, no. 5, pp. 1768-1778, 2015.

[12] T.-W. Yoo and K. Chang, "Theoretical and experimental development of 10 and $35 \mathrm{GHz}$ rectennas," IEEE Transactions on 
Microwave Theory and Techniques, vol. 40, no. 6, pp. 1259-1266, 1992.

[13] J. McSpadden, T. Yoo, and K. Chang, "Diode characterization in a microstrip measurement system for high power microwave power transmission," in Proceedings of the IEEE MTT-S International Microwave Symposium Digest Part 2 (of 3), pp. 1015-1018, IEEE, June 1992.

[14] Y.-J. Ren, M.-Y. Li, and K. Chang, " 35 GHz rectifying antenna for wireless power transmission," Electronics Letters, vol. 43, no. 11, pp. 602-603, 2007.

[15] H.-K. Chiou and I.-S. Chen, "High-efficiency dual-band onchip rectenna for 35 - and $94-\mathrm{GHz}$ wireless power transmission in $0.13-\mu \mathrm{m}$ CMOS technology," IEEE Transactions on Microwave Theory and Techniques, vol. 58, no. 12, pp. 35983606, 2010.

[16] S. Ladan and K. Wu, "Nonlinear modeling and harmonic recycling of millimeter-wave rectifier circuit," IEEE Transactions on Microwave Theory and Techniques, vol. 63, no. 3, pp. 937-944, 2015. 


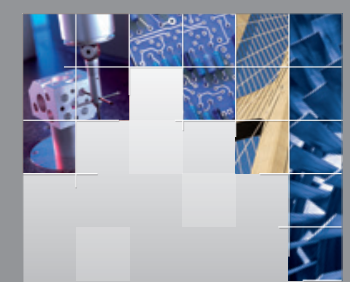

\section{Enfincering}
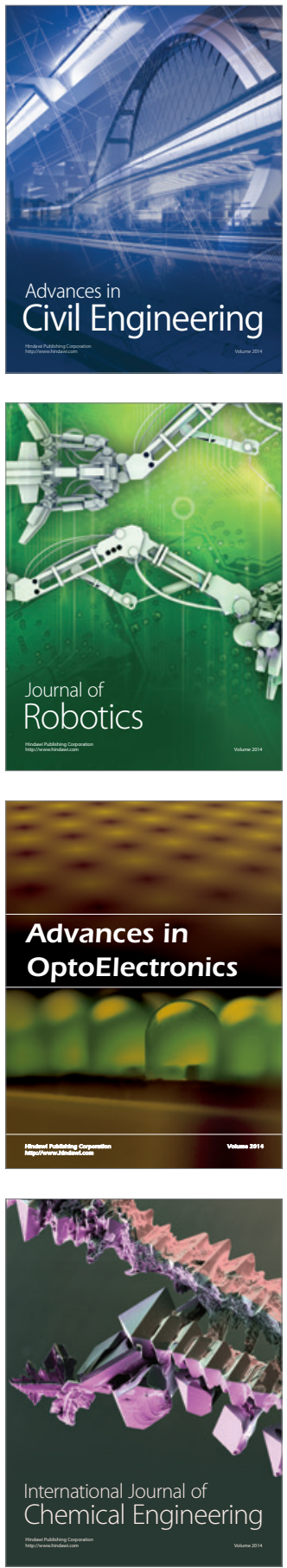

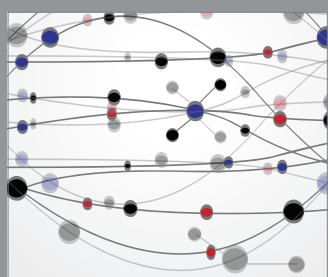

The Scientific World Journal

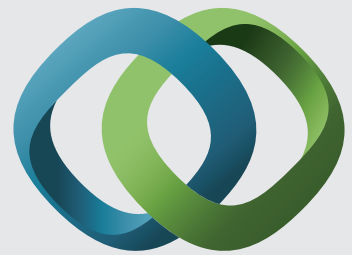

\section{Hindawi}

Submit your manuscripts at

http://www.hindawi.com
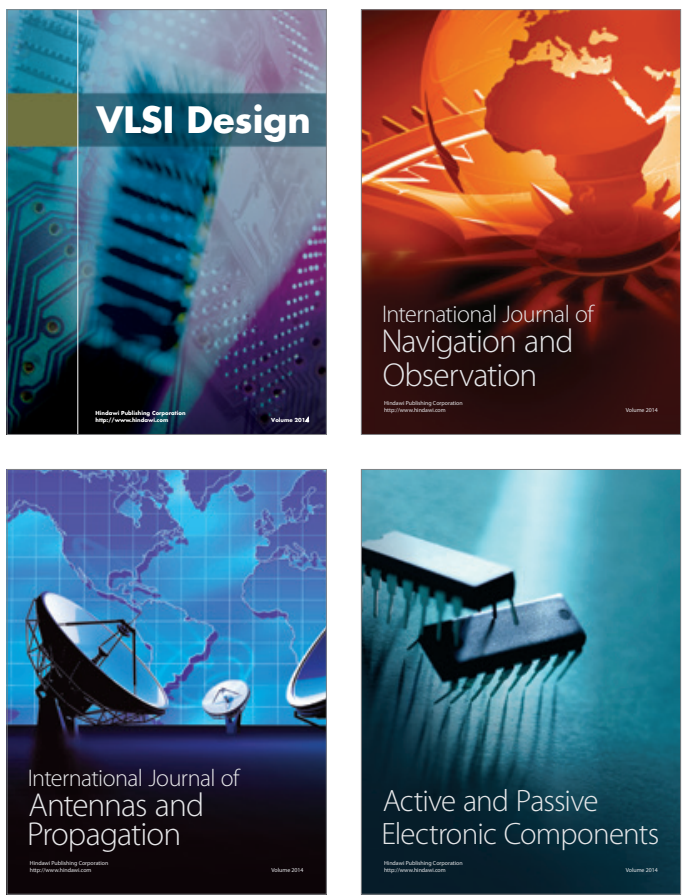
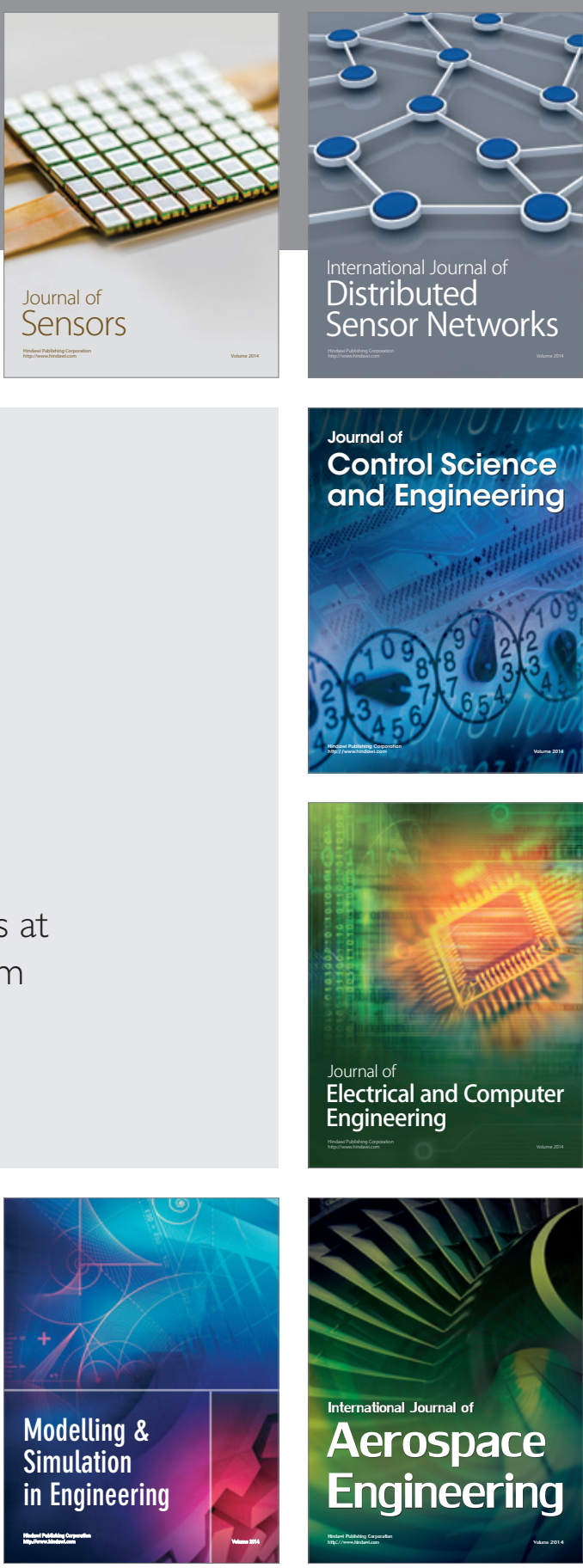

International Journal of

Distributed

Sensor Networks

Journal of

Control Science

and Engineering
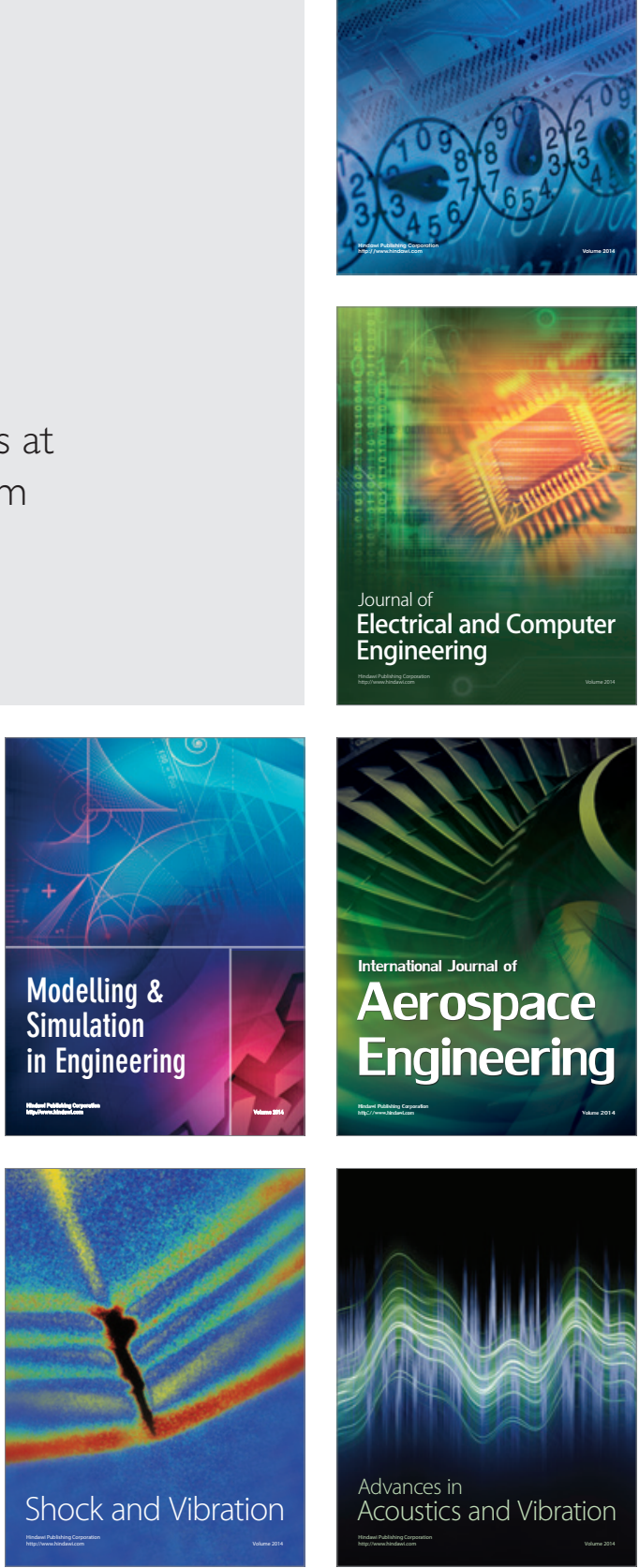